\title{
Constraining the Structure of GRB Jets Through the Afterglow Light Curves
}

\author{
Jonathan Granot ${ }^{1}$ and Pawan Kumar ${ }^{2}$
}

\begin{abstract}
We investigate the effect that the structure of GRB jets has on the afterglow light curves for observers located at different viewing angles, $\theta_{\text {obs }}$, from the jet symmetry axis. The largest uncertainty in the jet dynamics is the degree of lateral energy transfer. Thus, we use two simple models, that make opposite and extreme assumptions for this point, and calculate the light curves for an external density that is either homogeneous, or decreases as the square of the distance from the source. The Lorentz factor and kinetic energy per unit solid angle are initially taken to be power laws of the angle from the jet axis. We perform a qualitative comparison between the resulting light curves and afterglow observations. This constrains the jet structure, and poses problems for a 'universal' jet model, where all GRB jets are assumed to be intrinsically identical, and differ only by our viewing angle, $\theta_{\text {obs }}$.
\end{abstract}

Subject headings: gamma rays: bursts - gamma-rays: theory - shock waves relativity - radiation mechanisms: nonthermal

\section{Introduction}

There are several different lines of evidence in favor of collimated outflows, also referred to as jets, in gamma-ray bursts (GRBs). Perhaps, the best evidence so far is the achromatic break seen in the light curves of many (though not all) GRB afterglows. A collimated outflow also helps to reduce the estimate for the total energy output in gamma-rays that is inferred from the fluence in GRBs with known redshifts, which in some cases approaches and in one case (GRB 990123) even exceeds the rest energy of a solar mass star, for a spherically symmetric emission. Such an energy output in gamma-rays is hard to produce in any model that involves a stellar mass object. Furthermore, a non-spherical flow can also manifest itself

\footnotetext{
${ }^{1}$ Institute for Advanced Study, Olden Lane, Princeton, NJ 08540; granot@ias.edu

${ }^{2}$ Astronomy Department, University of Texas, Austin, TX 78731; pk@surya.as.utexas.edu
} 
through linear polarization (Sari 1999; Ghisellini \& Lazzati 1999) as was indeed observed for a few afterglows (Covino et al. 1999; Wijers et al. 1999; Rol et al. 2000).

Most GRB jet models consider an outflow that is uniform within some finite, well defined, opening angle around its symmetry axis, and where the Lorentz factor, energy density etc. drop sharply beyond this opening angle (Rhoads 1997, 1999; Panaitescu \& Mészáros 1999; Sari, Piran \& Halpern 1999; Kumar \& Panaitescu 2000; Moderski, Sikora \& Bulik 2000; Granot et al. 2001, 2002). Such a uniform jet with a sharp, well defined, edge shall be referred to as a 'top hat' jet. The possibility that GRB jets can display an angular structure, where the kinetic energy per unit solid angle, $\epsilon$, and the Lorentz factor, $\Gamma$, of the GRB outflow vary smoothly as power laws in the angle, $\theta$, from the jet axis, was proposed by Mészáros, Rees \& Wijers (1998). We shall refer to such smoothly varying relativistic outflows as 'structured' jets (as opposed to a 'top hat' jet, that has no inner structure).

Recently, several different groups have analyzed afterglow observations within the frame work of the 'top hat' jet model, and have inferred a relatively narrow distribution both for the total energy output in gamma-rays (Frail et al. 2001) and in the initial kinetic energy of the relativistic outflow (Panaitescu \& Kumar 2001; Piran et al. 2001). These results may alternatively be interpreted as GRB jets having a universal structure, which is intrinsically the same for all GRBs, and the observed differences between different GRBs are a result of different viewing angle, $\theta_{\text {obs }}$, w.r.t. the jet symmetry axis (Lipunov, Postnov \& Prokhorov 2001; Rossi, Lazzati \& Rees 2002; Zhang \& Mészáros 2002). Whereas in the 'top hat' jet interpretation, the jet break time, $t_{j}$, depends mainly on the initial opening angle of the jet, $\theta_{j}$ (and also has a smaller dependence on its energy per unit solid angle and on the external density, e.g. Sari, Piran \& Halpern 1999), in the universal 'structured' jet interpretation, $t_{j}$ depends mainly on the viewing angle, $\theta_{\text {obs }}$, and the light curve is similar to that for a 'top hat' jet with an opening angle $\theta_{j}=\theta_{\text {obs }}$ and the same value of the energy per unit solid angle of the 'structured' jet at $\theta=\theta_{\text {obs }}$.

While the evolution of 'top hat' jets and their light curves have been widely investigated (Rhoads 1999; Panaitescu \& Mészáros 1999; Kumar \& Panaitescu 2000; Moderski, Sikora \& Bulik 2000; Granot et al. 2002), including numerical simulations of the jet dynamics and calculation of the resulting light curves (Granot et al. 2001), much less work has been done on 'structured' jets. In an accompanying paper (Kumar \& Granot 2002) we calculate the dynamics of 'structured' relativistic jets by solving relativistic fluid dynamics equations, and demonstrate that a simple analytic or semi-analytic model can qualitatively reproduce the results for the jet dynamics and for the light curves as long as the Lorentz factor along the jet axis is of order 4 or larger. In this paper we use two simple models for the jet dynamics, which are designed to bracket the true hydrodynamic evolution of the jet, to calculate the 
afterglow light curves for a wide range of parameters for the structured jet and the external density. A qualitative comparison of the light curves with afterglow observations provides constraints on the jet structure and density profile in its immediate vicinity.

In $\S 2$ we present the physical model. We begin with the initial conditions (§2.1), then describe our two simple dynamical models (§2.2) and finally outline the procedure for calculating the light curves $(\S 2.3)$. Our results are presented in $\S 3$, and in $\S 4$ we discuss our main conclusions.

\section{The Physical model}

The jet is assumed to posses axial symmetry, so that all the hydrodynamic quantities at a given lab frame time, $t$, depend only on the angle, $\theta$, from the jet symmetry axis. The radial profile of the outflow is ignored in this simple treatment, and the shocked material is approximated by a thin shell that is located at the same radius, $R$, of the shock.

The observed afterglow light curves depend on the angular structure of the GRB jet, its hydrodynamic evolution, and the viewing angle, $\theta_{\text {obs }}$, w.r.t. the jet symmetry axis. One may therefore use the shape of the afterglow light curves to constrain the angular structure of the jet, as well as to infer the viewing angle, $\theta_{\text {obs }}$. For a 'top hat' jet of opening angle $\theta_{0}$, $\theta_{\text {obs }}<\theta_{0}$ is required in order to observe the prompt gamma-ray emission. In this case the differences in the light curves between different $\theta_{\text {obs }}$ in the range $0 \leq \theta_{\text {obs }}<\theta_{0}$ are rather small (Granot et al. 2001, 2002). However, for structured jets we expect large differences in lightcurves for different $\theta_{\text {obs }}$.

\subsection{The Initial Conditions}

The initial conditions are chosen at a lab frame time, $t_{0}$, for which on the one hand, the internal shocks have ended, and on the other hand, no significant deceleration due to the sweeping up of the external medium has yet occurred (i.e. $R_{I S}(\theta)<R\left(\theta, t_{0}\right)<R_{\text {dec }}(\theta)$ for all relevant $\theta$ ). For simplicity, the original ejecta is assumed to remain cold, even near the deceleration radius, where it is expected to be heated by the passage of the reverse shock. This approximation might introduce inaccuracies of order unity near the deceleration epoch, corresponding to an observed time $\lesssim$ a few minutes for a typical GRB, but it should have no effect on lightcurves at later times. Energy conservation implies

$$
\epsilon\left(\theta, t_{0}\right)=\mu_{0}\left(\theta, t_{0}\right)\left[\Gamma\left(\theta, t_{0}\right)-1\right]+\mu_{s}\left(\theta, t_{0}\right)\left[\Gamma^{2}\left(\theta, t_{0}\right)-1\right] \approx \mu_{0}\left(\theta, t_{0}\right) \Gamma\left(\theta, t_{0}\right)
$$


where $\mu_{0}$ and $\mu_{s}$ are the rest mass per unit solid angle of the original ejecta and of the swept up external medium, respectively, $\epsilon$ is the energy (not including the energy associated with the rest mass) per unit solid angle in the outflow, $\Gamma$ is the bulk Lorentz factor of the shocked material, and $t_{0}$ is chosen so that $\mu_{s}\left(\theta, t_{0}\right) \Gamma\left(\theta, t_{0}\right) \ll \mu_{0}\left(\theta, t_{0}\right)$ for all $\theta$.

The initial kinetic energy per unit solid angle, and initial Lorentz factor (minus 1) are assumed to be power laws in $\theta$, outside of a core of opening angle $\theta_{c}$ :

$$
\epsilon\left(\theta, t_{0}\right)=\epsilon_{0} \Theta^{-a} \quad, \quad \Gamma\left(\theta, t_{0}\right)=1+\left(\Gamma_{0}-1\right) \Theta^{-b},
$$

where $\epsilon_{0}$ and $\Gamma_{0}$ are the initial kinetic energy per unit solid angle and Lorentz factor at the jet axis, and

$$
\Theta \equiv \sqrt{1+\left(\frac{\theta}{\theta_{c}}\right)^{2}} \approx\left\{\begin{array}{ll}
1 & \theta \ll \theta_{c} \\
\theta / \theta_{c} & \theta \gg \theta_{c}
\end{array} .\right.
$$

The velocity is assumed to be in the radial direction, so that the initial radius is given by

$$
R\left(\theta, t_{0}\right)=t_{0} \sqrt{1-\Gamma^{-2}\left(\theta, t_{0}\right)}
$$

and the lateral transfer of matter can be neglected ${ }^{3}$. The external mass density profile is assumed to be a power law in the distance $r$ from the source, $\rho_{\text {ext }}(r)=A r^{-k}$. This implies

$$
\begin{aligned}
& \mu_{s}(\theta, t)=\int_{0}^{R(\theta, t)} r^{2} d r \rho_{e x t}(r)=\frac{A R(\theta, t)^{3-k}}{(3-k)} \\
& \mu_{0}(\theta, t)=\mu_{0}\left(\theta, t_{0}\right)=\frac{\epsilon_{0} \Theta^{b-a}}{\left(\Gamma_{0}-1\right)}-\left[\Gamma\left(\theta, t_{0}\right)+1\right] \mu_{s}\left(\theta, t_{0}\right) .
\end{aligned}
$$

\subsection{The Jet Dynamics}

The main uncertainty in the jet dynamics is the degree of lateral transport of energy, from small to large angles, $\theta$, w.r.t. the jet axis. We therefore make two alternative and extreme assumptions regarding this transport: in model $\mathbf{1}$ we assume that the energy per unit solid angle, $\epsilon$, does not change with time, $\epsilon(\theta, t)=\epsilon\left(\theta, t_{0}\right)$, while in model 2 we assume the maximal averaging of $\epsilon$ over the angle $\theta$ that is consistent with causality. This is done by averaging over the initial distribution of $\epsilon$,

$$
\bar{\epsilon}(\theta, t) \equiv \frac{1}{\left(\cos \theta_{-}-\cos \theta_{+}\right)} \int_{\theta_{-}}^{\theta_{+}} d \theta^{\prime} \sin \theta^{\prime} \epsilon\left(\theta^{\prime}, t_{0}\right),
$$

\footnotetext{
${ }^{3} \mathrm{~A}$ more rigorous treatment of the jet hydrodynamics (Kumar \& Granot 2002) shows that these assumptions are reasonable.
} 
where $\theta_{-}\left(\theta_{+}\right)$is the angle below (above) $\theta$ (i.e. $\theta_{-}<\theta<\theta_{+}$), to which a hypothetical sound wave that originated at $\theta$ at the initial time $t_{0}$ would have propagated to. Initially, $\theta_{-}\left(t_{0}\right)=\theta_{+}\left(t_{0}\right)=\theta$, and these angles subsequently evolve according to

$$
\frac{\partial \theta_{ \pm}}{\partial t}= \pm \frac{c_{s}\left(\theta_{ \pm}, t\right)}{\Gamma\left(\theta_{ \pm}, t\right) R\left(\theta_{ \pm}, t\right)} \quad, \quad c_{s}=\sqrt{\frac{\hat{\gamma}(\hat{\gamma}-1)[\Gamma-1]}{1+\hat{\gamma}[\Gamma-1]}}
$$

where $c_{s}$ is the local sound speed, and $\hat{\gamma}=(4 \Gamma+1) / 3 \Gamma$ is the adiabatic index. This simple local scheme does not, in general, conserve the total energy in the outflow. The global energy conservation is imposed by renormalizing $\bar{\epsilon}$,

$$
\epsilon(\theta, t)=\bar{\epsilon}(\theta, t) \frac{\int_{0}^{\pi / 2} d \theta^{\prime} \sin \theta^{\prime} \epsilon\left(\theta^{\prime}, t_{0}\right)}{\int_{0}^{\pi / 2} d \theta^{\prime} \sin \theta^{\prime} \bar{\epsilon}\left(\theta^{\prime}, t\right)} .
$$

For both models 1 and 2, the radius of the thin shell of matter changes $\operatorname{as}^{4}$

$$
\frac{\partial R(\theta, t)}{\partial t}=\sqrt{1-\Gamma^{-2}(\theta, t)}
$$

while $\mu_{s}$ and $\mu_{0}$ are given by equation (5) and (6), respectively, and the Lorentz factor of the shocked material, $\Gamma$, is obtained by solving the equation for energy conservation,

$$
\epsilon=(\Gamma-1) \mu_{0}+\left(\Gamma^{2}-1\right) \mu_{s} \quad, \quad \Gamma=\frac{\mu_{0}}{2 \mu_{s}}\left[\sqrt{1+\frac{4 \mu_{s}\left(\epsilon+\mu_{0}+\mu_{s}\right)}{\mu_{0}^{2}}}-1\right] .
$$

\subsection{The Light Curves}

The local emissivity is calculated using the conventional assumptions of synchrotron emission from relativistic electrons that are accelerated behind the shock into a power law distribution of energies, $N(\gamma) \propto \gamma^{-p}$ for $\gamma>\gamma_{m}$, where the electrons and the magnetic field hold fractions $\epsilon_{e}$ and $\epsilon_{B}$, respectively, of the internal energy. The shape of the local spectral emissivity is approximated as a broken power law with breaks at the typical synchrotron frequency $\nu_{m}$ and at the cooling frequency $\nu_{c}$ (Sari, Piran \& Narayan 1998). As our main focus in this work is the effect of the jet dynamics on the afterglow emission, we keep the expression for the local emissivity fairly simple, and leave the inclusion of additional features such as the self absorption frequency, inverse Compton emission and its effect on the electron

\footnotetext{
${ }^{4}$ There is a small difference between the velocity of the shock front and that of the fluid just behind the shock (Blandford \& McKee 1976), however we neglect this distinction in the present treatment, for simplicity.
} 
cooling, etc., for later applications, since such complications might make it hard to pinpoint the effects of the jet dynamics on the light curves.

The light curves for observers located at different angles, $\theta_{\text {obs }}$, w.r.t. the jet axis, are calculated by applying the appropriate relativistic transformation of the radiation field from the local rest frame of the emitting fluid to the observer frames, and integrating over equal

photon arrival time surfaces (Granot, Piran \& Sari 1999; Kumar \& Panaitescu 2000; Granot et al. 2001). The radiation calculation is in this sense rigorous and takes into account all relevant effects, so that the resulting light curves accurately reflect what is expected for a given jet structure and dynamics, where the latter are much less certain.

\section{Results}

In Figures 1-3 we show the evolution of the hydrodynamic quantities according to the two models described in the previous section, for a constant density external medium $(k=0)$. In Figure 1 we show the evolution of the Lorentz factor for $(a, b)=(0,2)$. For $a=0$, models 1 and 2 become identical, so that this figure applies to both models. Since $\epsilon(\theta, t)=\epsilon_{0}=$ const, the evolution after the deceleration time is the same for all $\theta$, and different $\theta$ differ just by the deceleration time, $t_{\mathrm{dec}}$, and the corresponding deceleration radius, $R_{\mathrm{dec}}$, that are given by $\mu_{s} \sim \mu_{0} / \Gamma \sim \epsilon / \Gamma^{2}$, or

$$
R_{\mathrm{dec}}=\left[\frac{(3-k) \epsilon_{0}}{A c^{2} \Gamma_{0}^{2}}\right]^{\frac{1}{(3-k)}} \Theta^{\frac{2 b-a}{(3-k)}} \quad, \quad t_{\mathrm{dec}}=\frac{R_{\mathrm{dec}}}{2 c \Gamma^{2}\left(t_{0}\right)} \propto \Theta^{\frac{2 b(4-k)-a}{(3-k)}}
$$

As can be seen from equation (12), for $a=0$, the deceleration occurs first at small $\theta$, and gradually proceeds to larger angles. This can be nicely seen in Figure 1.

This situation is similar for $(a, b)=(2,2)$, but is reversed for $(a, b)=(2,0)$, as can be seen in Figures 2 and 3, where in the latter case the deceleration occurs first at large angles $\theta$ and then proceeds to smaller angles. This is in agreement with equation (12). The upper panel of Figures 2 and 3 shows the energy per unit solid angle, $\epsilon$, which is initially the same for the two models, and remains unchanged for model 1, while for model $2 \epsilon$ decreases at small angles $\theta$, and increases at large angles. This indicates a lateral transfer of energy from small to large angles. At late times, as the flow becomes sub-relativistic, $\epsilon$ becomes almost independent of angle $\theta$, and the flow approaches spherical symmetry, as is indeed expected to occur physically, since the spherically symmetric Sedov-Taylor self similar solution should be asymptotically approached in the non-relativistic regime. The middle and bottom panels show the Lorentz factor minus one, $\Gamma-1$, for models 1 and 2, respectively. For model 1 , it can be seen that while the flow is still relativistic, The Lorentz factor soon settles into 
a profile of $\Gamma-1 \propto \theta^{-1}$ at $\theta>\theta_{c}$, instead of the initial $\Gamma-1 \propto \theta^{-2}$ or $\Gamma=$ const. This occurs since the fraction of the energy in the original ejecta soon becomes negligible implying $\epsilon \approx \mu_{s}\left(\Gamma^{2}-1\right)$ so that as long as the flow is relativistic, $\mu_{s} \propto R^{3-k} \approx$ const, since $R \approx c t$ for all $\theta$, and $\Gamma-1 \propto \epsilon^{1 / 2} \propto \theta^{-1}$. When the flow becomes non-relativistic, $\Gamma^{2}-1 \approx 2(\Gamma-1) \approx \beta^{2}$ so that $\Gamma-1 \propto \theta^{-4 /(5-k)}$, which is still quite close to $\theta^{-1}$ for $k$ between 0 and 2 . For model 2 , a similar effect is seen at early times, when $\epsilon(\theta, t) \approx \epsilon\left(\theta, t_{0}\right)$, but it becomes much more homogeneous at later times, as $\epsilon(\theta)$ becomes more uniform.

The light curves of models 1 and 2 for $(a, b)=(0,0),(0,2),(2,0),(2,2)$ and a uniform external medium $(k=0)$ are shown in Figure 4 , while the temporal decay slope, $\alpha$ (defined by $\left.F_{\nu} \propto t^{\alpha}\right)$, for the same light curves, is shown in Figure 5 . We have added the spherical case, $(a, b)=(0,0)$, for comparison. For $(a, b)=(0,2)$, the light curve initially rises, before the deceleration time, $t_{\text {dec }}$, which occurs at latter times for larger viewing angles $\theta_{\text {obs }}$, in accord with equation (12), when keeping in mind that the emission is dominated by $\theta \sim \theta_{\text {obs }}$ at early times due to the relativistic beaming of the radiation emitted from the jet. After a time $t$, deceleration has occurred at $\theta<\theta_{\operatorname{dec}}(t)$, where $\theta_{\text {dec }}$ is given by $t_{\operatorname{dec}}\left[\theta_{\operatorname{dec}}(t)\right]=t$, and the light curves for $\theta_{\text {obs }}<\theta_{\text {dec }}(t)$ approach the spherical case, and hence become very close to one other. Keeping $a=0, \epsilon$ and $\Gamma_{0}$ fixed, and increasing $b$ from zero, $t_{\text {dec }}\left(\theta_{\text {obs }}\right)$ begins to shift to larger times, and the ratio of the deceleration times for two given values of $\theta_{\text {obs }}$ grows as $b$ increases. But after the time $t_{\text {dec }}\left(\theta_{\text {obs }}\right)$, the light curves still approach the same spherical light curve, for all values of $b$. There is no jet break in the light curve for $a=0$. Since jet breaks are observed in many afterglows, this type of jet structure can be ruled out as a universal model for GRB jets.

For $(a, b)=(2,0)$ and $(2,2)$ we find a jet break in the light curve at roughly the same time as predicted in previous works (Rossi, Lazzati \& Rees 2002; Zhang \& Mészáros 2002), i.e. when $\Gamma\left(\theta=\theta_{\text {obs }}\right) \sim \theta_{\text {obs }}^{-1}$. However, we also find some new and interesting features. For $(a, b)=(2,2)$ with $\Gamma_{0} \lesssim 10^{3}$, the initial Lorentz factor at large angles, $\theta$, is quite modest, resulting in a large deceleration time, $t_{\mathrm{dec}}$, which can be as large as $t_{\mathrm{dec}} \gtrsim 1$ day for $\theta_{\text {obs }} \gtrsim 0.2$. This would result in a rising light curve at early times, for all frequencies. On the other hand, for $(a, b)=(2,0)$, where the initial Lorentz factor is the same for all $\theta$, the deceleration time, $t_{\text {dec }}$, is very small everywhere, so that the initial rise of the light curve at $t<t_{\text {dec }}$ will be very hard to observe. The lack of an observation of a rising light curve for afterglow observations starting from a few hours after the burst can already constrain the jet structure: either $b<2$ or $\Gamma_{0}>10^{3}$ are required. Future observations at much earlier times after the burst, as may be achieved with the forthcoming Swift mission, may provide much stronger constraints on the jet structure.

It can also be seen from Figure 4 that for $(a, b)=(2,2)$, the value of the temporal decay 
slope, $\alpha$ (defined by $F_{\nu} \propto t^{\alpha}$ ), before the jet break, is higher for larger viewing angles $\theta_{\text {obs }}$. This effect is very large for model 1 , but not very significant for model 2 (see Figure 5 ). The value of $\alpha$ before the break in the light curve for model 1 is larger than the observed value for several well studied GRB afterglows, and the light curve power-law steepening, $|\delta \alpha|$, is smaller than the observed value for many GRBs. The light curves obtained using model '1' at early times are almost identical to the light curves from hydrodynamical simulations, and this suggests that jet structure described by $(a, b)=(2,2)$ is not consistent with the observations for a number of GRBs.

For $(a, b)=(2,0)$ the correlation between $\alpha$ before the jet break and $\theta_{\text {obs }}$ is not seen, but instead there is a flattening of the light curve (i.e. an increase in $\alpha$ ) just before the jet break, for viewing angles sufficiently large compared to the core angle of the jet, $\theta_{\text {obs }} \gtrsim 3 \theta_{c}$. This effect is more prominent in model 1, where it can also be seen to a lesser extent for $(a, b)=(2,2)$ as well, compared to model 2 , where this effect is smaller and can be seen only for $(a, b)=(2,0)$ (see Figure 5 ). Light curves calculated for the same jet profiles but with the jet dynamics given by a hydro-simulation (Kumar \& Granot 2002) show that for $(a, b)=(2,0)$ a reasonably sharp jet break is obtained only for $\theta_{\text {obs }} \gtrsim(2-3) \theta_{c}$, while the flattening in the light curve just before the jet break becomes prominent at $\theta_{\text {obs }} \gtrsim(5-7) \theta_{c}$. This leaves only a factor of $\sim 3$ in $\theta_{\text {obs }} / \theta_{c}$ for which there is a sharp jet break with no flattening in the light curve just before this break, as is seen in all afterglows with jet breaks. However, the inferred values of $\theta_{\text {obs }}$ range at least one order of magnitude, $\theta_{\text {obs }} \sim 2-20^{\circ}$. Therefore, $(a, b)=(2,0)$ is unlikely to be a universal jet profile, for all GRBs, since $\theta_{c}$ is expected to vary between different afterglows. Even without requiring a universal jet profile, i.e. the same $\theta_{c}$ for all GRB jets, it would be highly improbable if all viewing angles would by chance fall within the narrow range that gives a light curve qualitatively similar to observations. This can exclude $(a, b)=(2,0)$ for most GRBs, and imply $b>0$ (or even $b \gtrsim 1)$. It is interesting to note that this provides a lower limit on $b$, while the lack of detection of a rising light curve at early times may provide an upper limit on $b$. This may eventually pin down the value of $b$, or alternatively, call into question the simple jet structure interpretation discussed in this paper if the data is inconsistent with a reasonable value for $b$.

Figure 6 shows the light curves for an external density that drops as $r^{-2}(k=2)$. This corresponds to a stellar wind of a massive star progenitor. In the pulsar wind bubble model of GRBs, the effective external density can have different profile which may be approximated as a power law with an index $k$ that ranges between 0 and 1 (Königl \& Granot 2002). Therefore $k=0$ and $k=2$, are the extreme values of $k$ that may be expected, and an intermediate value of $k$, resulting in an intermediate behavior of the light curves, is possible. 
For $k=2$ we see a break in the light curve for a spherical flow $(a=b=0)$, at $t \sim 10 \mathrm{yr}$, corresponding to the non-relativistic transition time, $t_{\mathrm{NR}}$. This can also be seen in Figure 7, which shows the temporal decay slope, $\alpha$, for the same light curves shown in Figure 6 . Generally, $t_{\mathrm{NR}} \propto E^{1 /(3-k)}$, so that for $k=2$ it is linear in $E$, and a more moderate total energy of, say, $10^{51} \mathrm{erg}$ would give $t_{\mathrm{NR}} \sim 1 \mathrm{yr}$. We extend the observer time for which we show the light curves in order to illustrate that in the non-relativistic regime, all light curves become similar. One should keep in mind, that physically, one may expect an $r^{-2}$ external density profile only up to some finite radius (for a stellar wind, this correspond to the radius of the wind termination shock) while at much larger radii we expect a roughly constant density medium. This would result in modifications to the light curves shown here, however, this is typically expected to effect the light curves only in the non-relativistic regime $\left(t \gtrsim t_{\mathrm{NR}}\right)$.

The deceleration time, $t_{\mathrm{dec}}$, is typically very small for $k=2$ since the density at small radii is very large, and the total swept up mass is only linear in radius. The jet break is much smoother and less sharp compared to a constant density environment $(k=0)$, as was found for a 'top hat' jet considered by Kumar \& Panaitescu (2000). There is a relatively sharp break only for $(a, b)=(2,0)$, and even then it is hardly sharp enough to reproduce the jet breaks observed in GRB afterglows. This suggests $k<2$, or rather $k \lesssim 1$. It is interesting to note that this is consistent with the external density profile expected in the pulsar wind bubble model (Kónigl \& Granot 2002; Guetta \& Granot 2002), while this is not consistent with the density profile expected in the collapsar model, $k=2$.

\section{Discussion}

We have calculated light curves from structured relativistic jets (jets whose Lorentz factor and energy per unit solid angle vary smoothly with angle from the jet axis) using two simple models for the jet dynamics, motivated by the hydrodynamical simulation of Kumar and Granot (2002), and as two limiting cases of energy redistribution in the lateral direction.

The first model considers the energy per unit solid angle, $\epsilon(\theta, t)$, to be time independent, so that each segment of the jet evolves independently of the other parts of the jet, as if it was part of a spherical flow. This is roughly consistent with the results from hydrodynamical simulations of structured jets for which $\epsilon$ varies slowly with $\theta$, where we find the transverse velocity in the comoving frame to be small compared to the sound speed throughout much of the jet evolution, as long as the Lorentz factor, $\Gamma$, is of order a few or larger along the jet axis (Kumar \& Granot 2002). This model is expected to be a good approximation for calculating the jet dynamics and the resulting afterglow light curves for the first few days 
after a GRB.

The second model considers the maximum possible re-distribution of energy in the transverse direction so as to reduce the lateral-gradient of $\epsilon$. The energy per unit solid angle, $\epsilon(\theta, t)$, is taken to be proportional to the average over the initial distribution of $\epsilon, \epsilon\left(\theta, t_{0}\right)$, within the area out to which a sound wave could have traveled from $\theta$ since the initial time, $t_{0}$. This model is likely to be more accurate in describing the late time behavior of the jet and the light curves, when the transverse velocity becomes of order the sound speed.

We have calculated light-curves for a number of different initial jet structures, which are taken to be power-law profiles at angles larger than some core angle, i.e. $\epsilon \propto \theta^{-a}$ and $\Gamma \propto \theta^{-b}$ for $\theta>\theta_{c}$. We have considered three different jet profiles, namely $(a, b)=(2,0)$, $(2,2),(0,2)$ for both a homogeneous external medium as well an ambient density falling off as distance squared from the center of explosion (i.e. $k=0$ or 2 , where $\rho_{\text {ext }} \propto r^{-k}$ ).

The quantitative differences in the light-curves calculated using our two models are typically of order unity for all of the jet structures we have considered. This gives us some confidence that lightcurves can be calculated with a reasonable accuracy even with a crude modeling of jet dynamics. There are, however, interesting qualitative differences in the lightcurve properties depending on how we model jet dynamics. For instance, for $(a, b)=(2,2)$ and a homogeneous medium $(k=0)$, the temporal index of the light curve, $\alpha$ (defined by $\left.F_{\nu} \propto t^{\alpha}\right)$, before the jet break is larger for large viewing angles, $\theta_{\text {obs. }}$. This effect is much more prominent for model 1 , compared to model 2 . For $(a, b)=(2,0)$ and $k=0$, with model 1 there is a pronounced flattening in the light curve just before the jet break for $\theta_{\text {obs }} \gtrsim 3 \theta_{c}$, while for model 2 this effect is much smaller.

There is a significant difference between the two models in the late time light curves, as we expect: model 1 light curves continue a power-law decline at late times, whereas model 2 light curves show a slight flattening at late times resulting from energy redistribution - see Figures 4 and 5 for homogeneous external medium with jet structures $(a, b)=(2,2) \&(2,0)$, and Figures 6 and 7 for the stratified external medium the particular case of $(a, b)=(2,0)$. Figures 6 and 7 also show that the break in the light curves for a stratified external medium is very gentle and the change to the power-law index $\alpha$ is small so long as the observer is not too far the axis: $\theta_{\text {obs }} / \theta_{c} \lesssim 10$. This result is consistent with the work of Kumar \& Panaitescu (2000), based on the analysis of a 'top hat' jet model, that it is very difficult to see a break in light curves for jets in an $r^{-2}$ medium.

We can learn about the jet structure in GRBs from comparing our theoretical light curves to GRB afterglow observations. For instance, the light curves for $(a, b)=(2,0)$ and $(2,2)$ behave very differently prior to the jet break. This can be used to discriminate between 
these possibilities (Figures $4 \& 5$ ). For obvious reasons, the light curves for the $(0,2)$ case are very distinct and similar to a spherically symmetric explosion, and do not show a jet break. This jet structure can therefore be ruled out for all GRBs that show a jet break in their afterglow light curves. Clear breaks in the light curves can be seen for a stratified external medium density only when the observer is located far away from the jet axis $\left(\theta_{\text {obs }} \gtrsim 10 \theta_{c}\right)$, however, in this case the GRB will be faint and is likely to be missed in flux limited triggers for detecting GRBs.

The light curves we obtain for structured jets, in many cases show a different qualitative behavior compared to afterglow observations. For example, $k=2$ does not produce a sufficiently sharp jet break, while for $k=0$ only $a \approx 2$ produces jet breaks that resemble afterglow observations, and even then, it is very difficult to produce all current afterglow observations with a universal jet profile, that is, if all GRB jets have the same structure and differ only by our viewing angle, $\theta_{\text {obs }}$, w.r.t. the jet symmetry axis. Even if GRB jets do not have a universal structure, a comparison between the observed light curve and theoretical calculations can constrain the jet structure of each GRB separately.

Future afterglow observations should enable us to determine the structure of relativistic jets in GRBs as well as the properties of the surrounding medium. This will enable us to determine if the energy release in GRBs is nearly constant or not, and whether the observed afterglow light curves and the jet break time are determined by the observer angle w.r.t. a structured jet, or by the opening angle of a 'top hat' jet.

This work was supported by the Institute for Advanced Study, funds for natural sciences (JG).

\section{REFERENCES}

Covino, S., et al. 1999, A\&A, 348, L1

Frail, D. et al., 2001, ApJ, 562, L55

Ghisellini, G., \& Lazzati, D. 1999, A\&A, 309, L7

Granot, J., Piran, T., \& Sari, R. 1999, ApJ, 527, 236

Granot, J., et al. 2001, in "GRBs in the Afterglow Era", eds. E. Costa, F. Frontera \& J. Hjorth, Springer-Verlag:Berlin, 312

Granot, J., Panaitescu, A., Kumar, P., \& Woosley, S. E. 2002, ApJ, 570, L61 
Kumar, P., \& Granot, J. 2002, submitted to ApJ

Kumar, P., \& Panaitescu, A. 2000, ApJ, 541, L9

Lipunov, V.M., Postnov, K.A., \& Prokhorov, M.E. 2001, Astron. Rep., 45, 236

Mészáros, P., Rees, M.J, \& Wijers, R.A.M.J. 1998, ApJ, 499, 301

Moderski, R., Sikora, M., \& Bulik T. 2000, ApJ, 529, 151

Panaitescu, A., \& Kumar, P. 2002, ApJ, 560, L49

Panaitescu, A., \& Mészáros, P. 1999, ApJ, 526, 707

Piran, T., Panaitescu, A., Kumar, P., \& Piro, L. 2001, ApJ, 560, L167

Rhoads, J. 1997, ApJ, 487, L1

Rhoads, J. 1999, ApJ, 525, 737

Rol, E., et al. 2000, ApJ, 544, 707

Rossi, E., Lazzati, D., \& Rees, M.J. 2002, MNRAS, 332, 945

Sari, R. 1999, ApJ, 524, L43

Sari, R., Piran, T., \& Halpern, J. P. 1999, ApJ, 519, L17

Wijers, R.A.M.J., et al. 1999, ApJ, 523, L33

Zhang, B., \& Mészáros, P. 2002, ApJ, 571, 876 


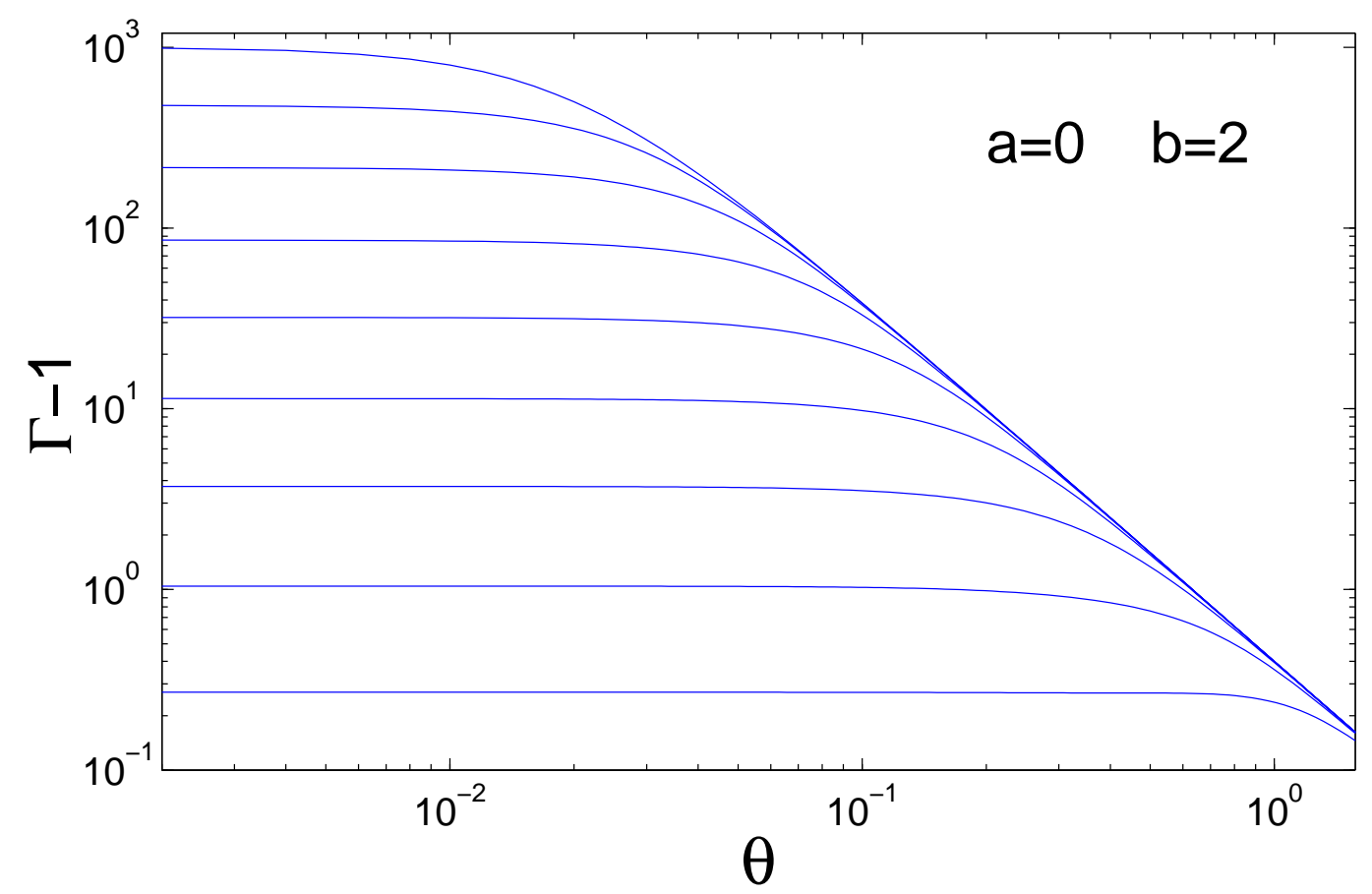

Fig. 1.- The evolution of the Lorentz factor $\Gamma(\theta)-1$, for $(a, b)=(0,2), \Gamma_{0}=10^{3}$ and $\theta_{c}=0.02 \mathrm{rad}$. For $a=0$, models 1 and 2 become the same, so that this figure applies to both models. 

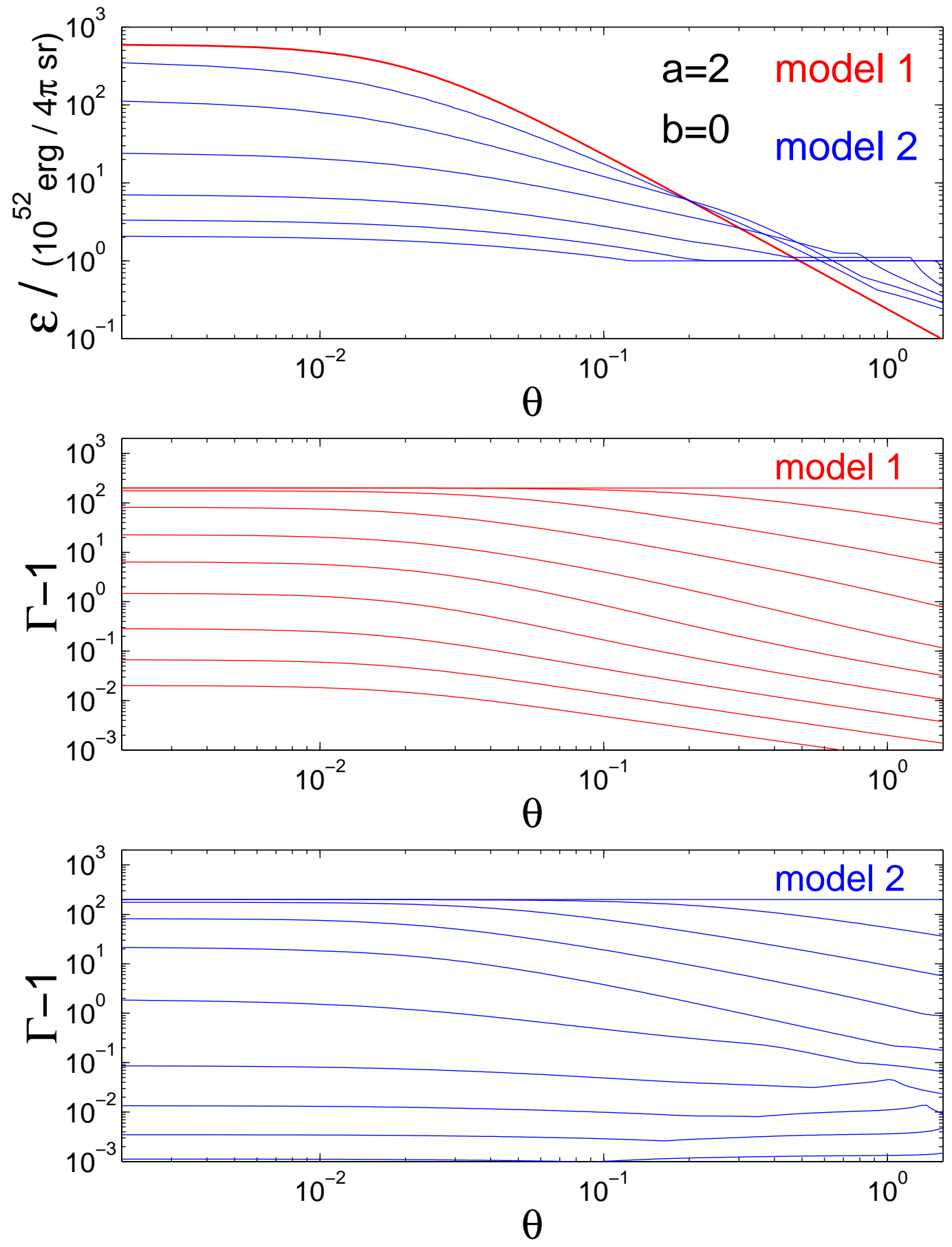

Fig. 2.- The hydrodynamic evolution of the energy per unit solid angle $\epsilon$ and the Lorentz factor $\Gamma-1$, as a function of the angle $\theta$ from the jet symmetry axis, according to our two simple and extreme models, for $(a, b)=(2,0)$. 

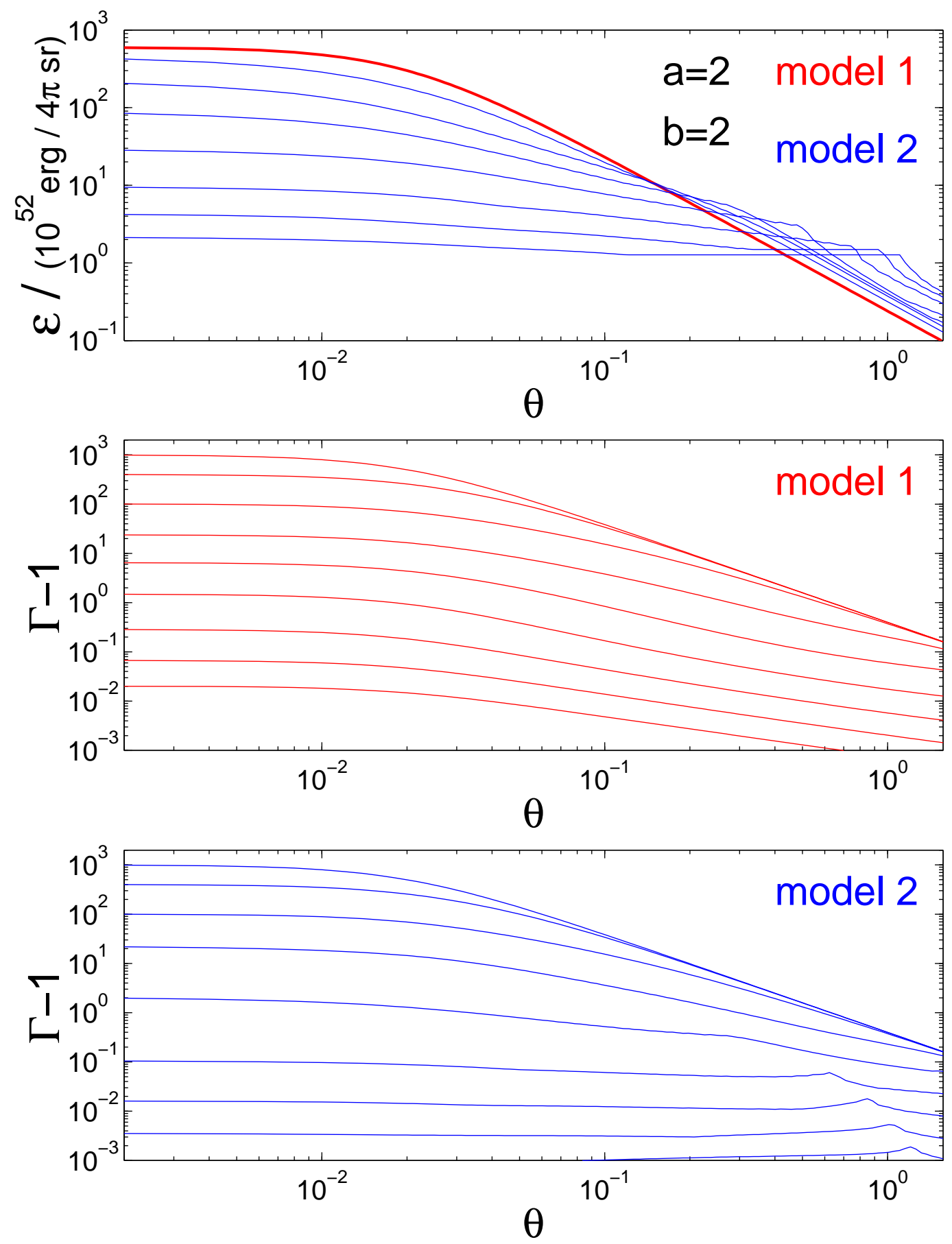

Fig. 3.- The same as figure 3 but for $(a, b)=(2,2)$. 

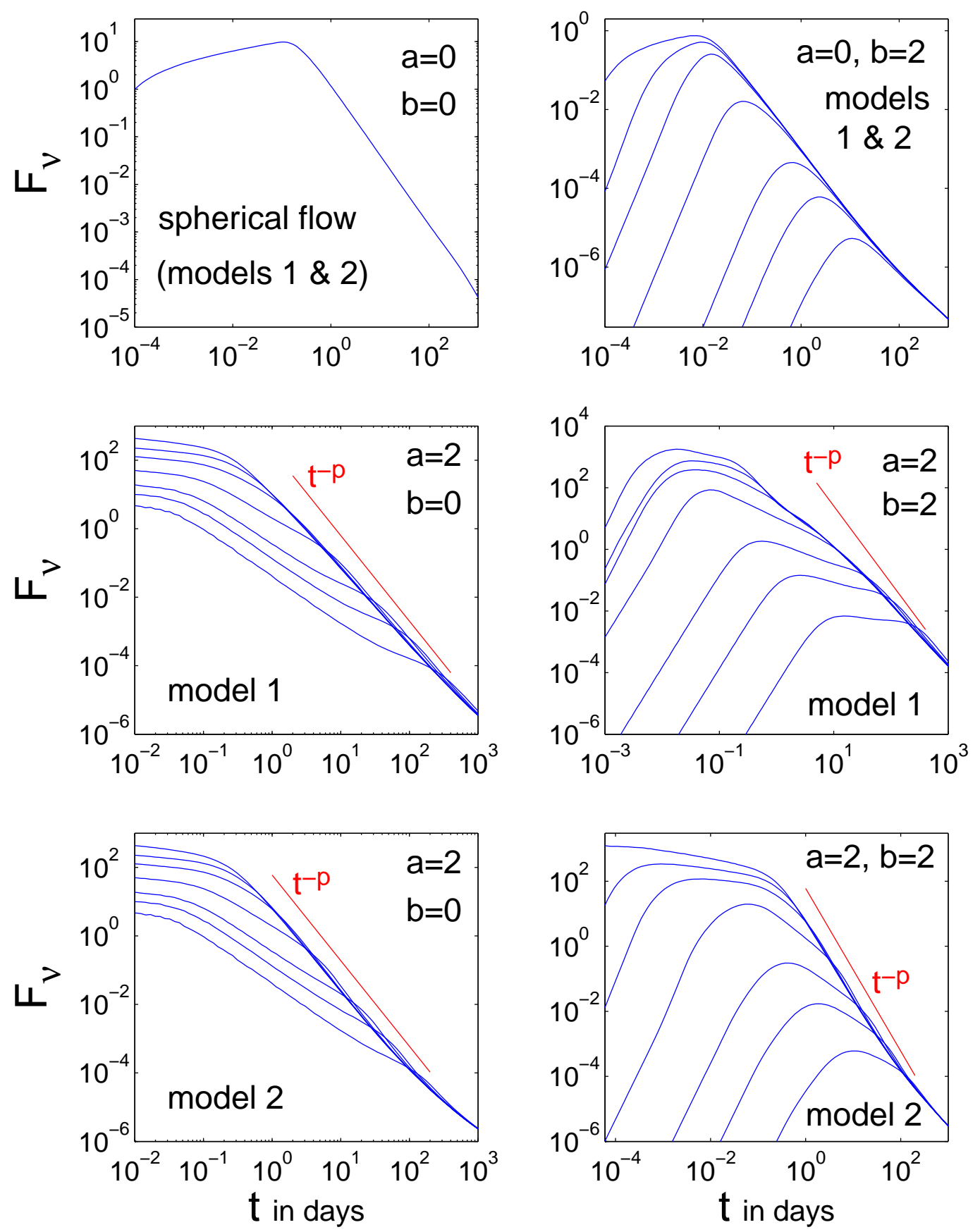

Fig. 4. - Light curves for a constant density external medium $(k=0)$, for models 1 and 2 (see text for details), in the optical $\left(\nu=5 \times 10^{14} \mathrm{~Hz}\right)$, for a jet core angle $\theta_{c}=0.02$, viewing angles $\theta_{\text {obs }}=0.01,0.03,0.05,0.1,0.2,0.3,0.5, p=2.5, \epsilon_{e}=\epsilon_{B}=0.1, n=1 \mathrm{~cm}^{-3}, \Gamma_{0}=10^{3}$, and $\epsilon_{0}$ was chosen so that the total energy of the jet would be $10^{52} \mathrm{erg}$. A power law of $t^{-p}$ is added in some of the panels, for comparison. 

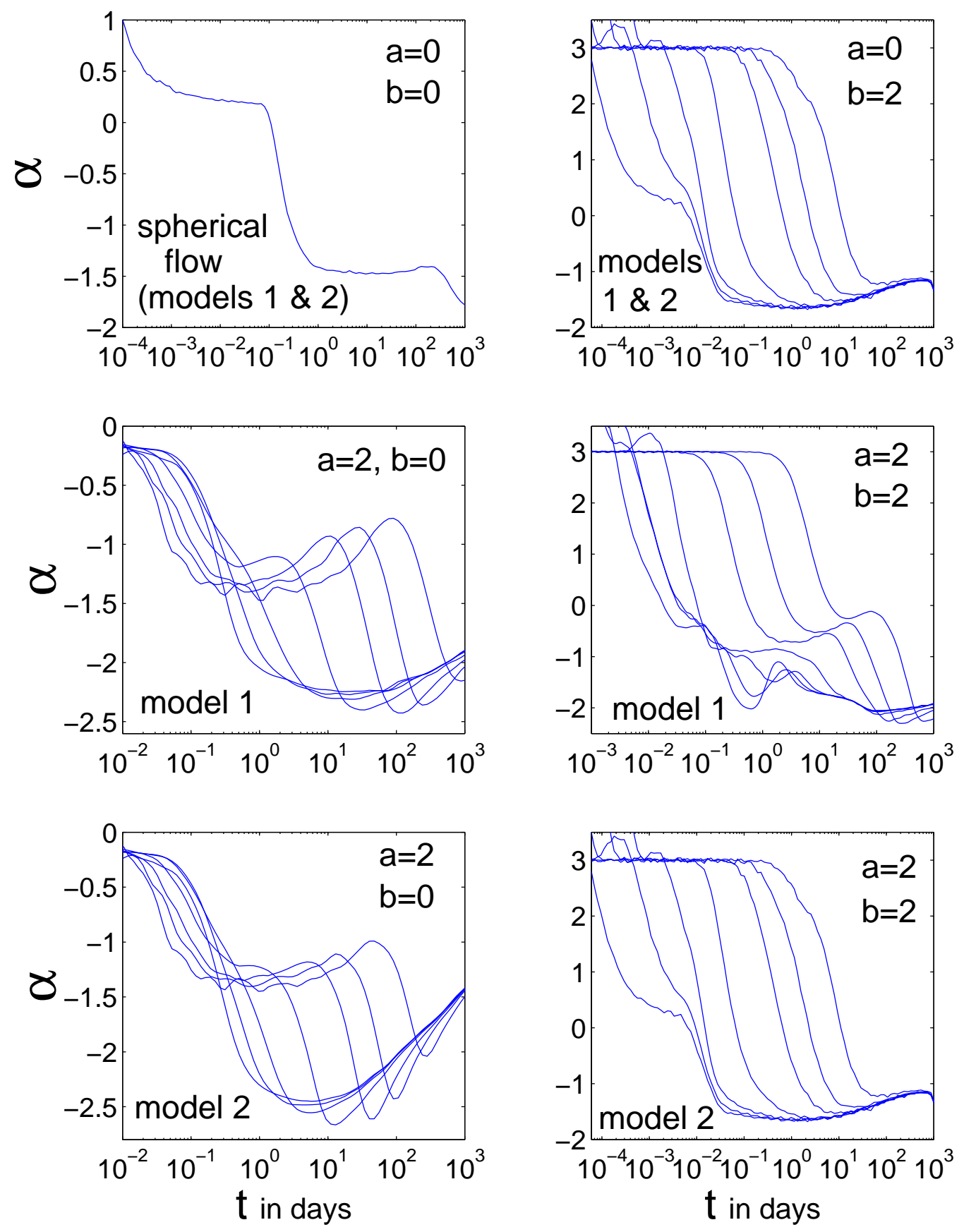

Fig. 5.- The temporal decay slope, $\alpha \equiv d \ln F_{\nu} / d \ln t$ (i.e. $F_{\nu} \propto t^{\alpha}$ ), for the light curves shown in figure 4 , that are with a constant density environment $(k=0)$. 

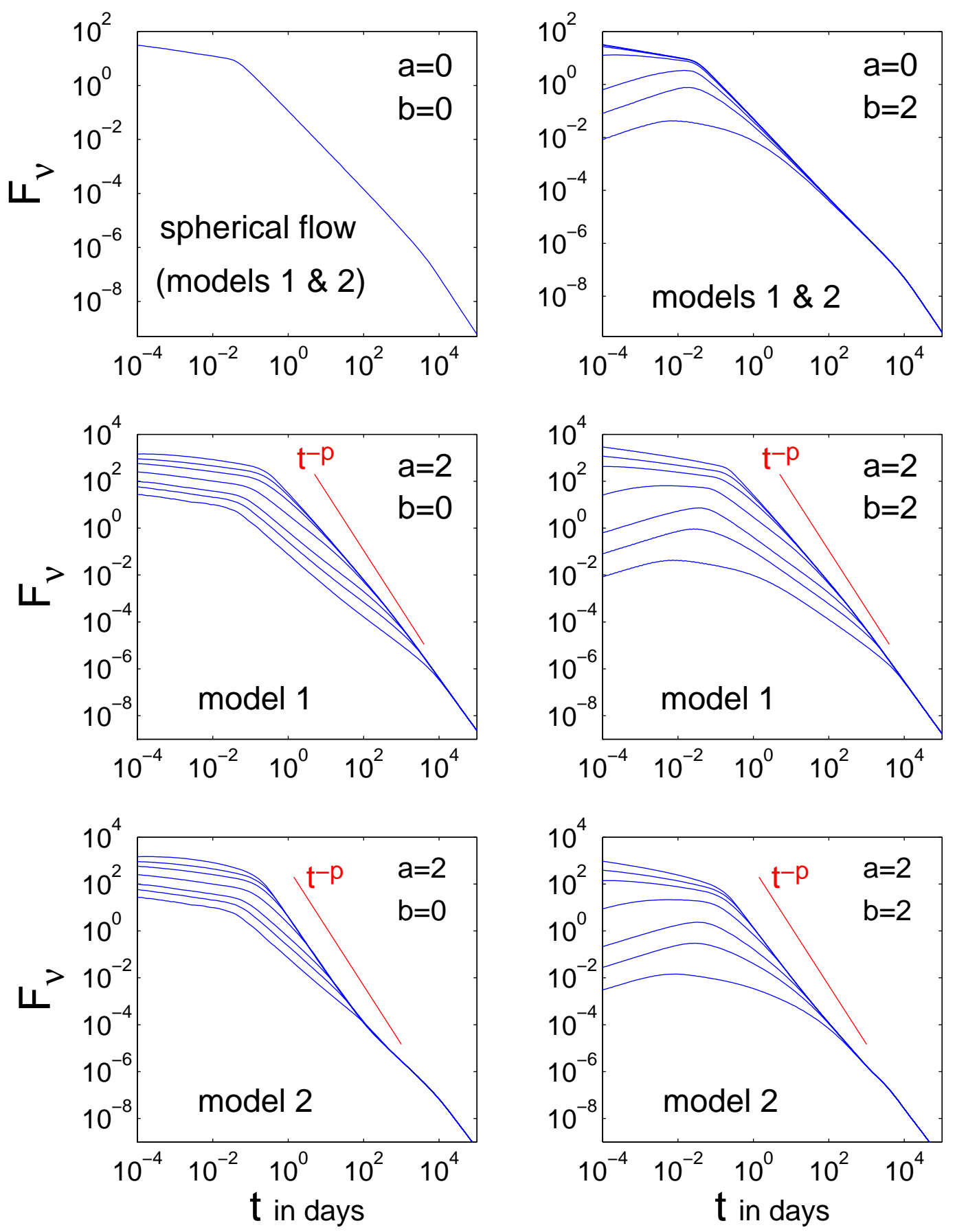

Fig. 6. - The same as Figure 4, but for an external density $\propto r^{-2}$, corresponding to a stellar wind, with $A=5 \times 10^{11} \mathrm{gr} \mathrm{cm}^{-1}$. 

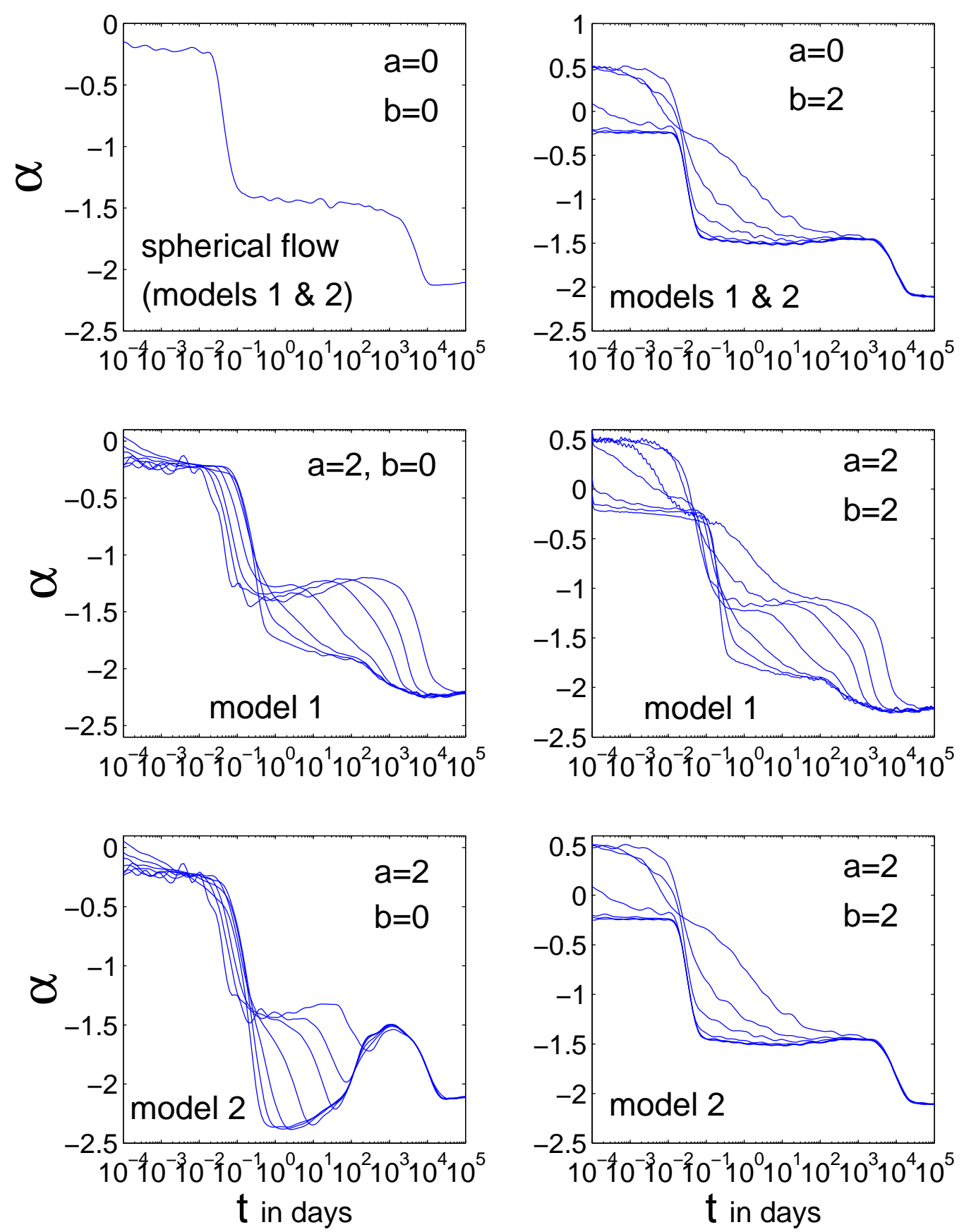

Fig. 7.- The temporal decay slope, $\alpha$, for the light curves shown in figure 6 , that are for a stellar wind environment $(k=2)$. 